\title{
Das Projekt „Erhaltungskulturen von bedrohten Pflanzen in Hessen“
}

\author{
Uwe Barth \& Andreas König
}

\begin{abstract}
As part of a conservation project supported by the KfW Foundation, 15 endangered plant species were collected and propagated in the Botanical Garden of the City of Frankfurt. Reintroduction activities have been carried out and documented, in cooperation with the nature conservation authorities in charge and local representatives of nature conservation associations. The endangered species comprise Allium strictum, Festuca albensis (syn. F. tomanii, F. duvalii p.p.), Fumana procumbens, Iris spuria, Jurinea cyanoides, Mibora minima, Moenchia erecta, Nigella arvensis, Poa badensis, Scleranthus verticillatus, Sedum villosum, Spergula pentandra, Ventenata dubia, Veronica acinifolia and Vicia orobus. All these are „species for which we have a special responsibility“ according to the Hessian biodiversity strategy. Two species, Mibora minima (Early Sand Grass) and Nigella arvensis, are described in more detail. The paper provides insights into details of seed collection, cultivation and re-application of native wild plants.
\end{abstract}

\section{Zusammenfassung}

Im Rahmen eines von der KfW Stiftung geförderten Artenschutzprojektes wurden Samen von 15 vom Aussterben bedrohten Pflanzenarten gesammelt und im Botanischen Garten der Stadt Frankfurt vermehrt. In Zusammenarbeit mit den zuständigen Naturschutzbehörden und lokalen Vertretern von Naturschutzverbänden wurden dokumentierte Wiederansiedlungsmaßnahmen durchgeführt. Bei den 15 gefährdeten Arten handelt es sich um Allium strictum, Festuca albensis (syn. F. tomanii, F. duvalii p.p.), Fumana procumbens, Iris spuria, Jurinea cyanoides, Mibora minima, Moenchia erecta, Nigella arvensis, Poa badensis, Scleranthus verticillatus, Sedum villosum, Spergula pentandra, Ventenata dubia, Veronica acinifolia, Vicia orobus. Alle 15 Arten sind als „Verantwortungsarten" Bestandteil der hessischen Biodiversitätsstrategie. Zwei dieser Arten, Mibora minima (Sand-Zwerggras) und Nigella arvensis (Acker-Schwarzkümmel) werden näher vorgestellt. Der Beitrag gibt anhand von Beispielen einen Einblick in die praktische Durchführung der Samensammlung, Kultivierung und Wiederausbringung heimischer Wildpflanzen.

\section{Einführung}

In letzter Zeit konnte wiederholt beobachtet werden, dass im Botanischen Garten der Dienstwagen mit Paletten junger Pflänzchen beladen wurde, oftmals bis unters Dach, dazu Kanister mit Wasser, Werkzeuge und Gießkannen. Dazu sah man einen Trupp Gärtner damit davon fahren (Abb. 1). Zuvor wurden diese Pflanzen, allesamt kleine unscheinbare Kräuter, in der Anzuchtsgärtnerei aus eigens dafür beschafftem Saatgut angezogen (Abb. 2). Gefragt, wohin die Reise denn gehe, hieß es dann „Schwanheimer Düne“ oder „Ober-Mör-

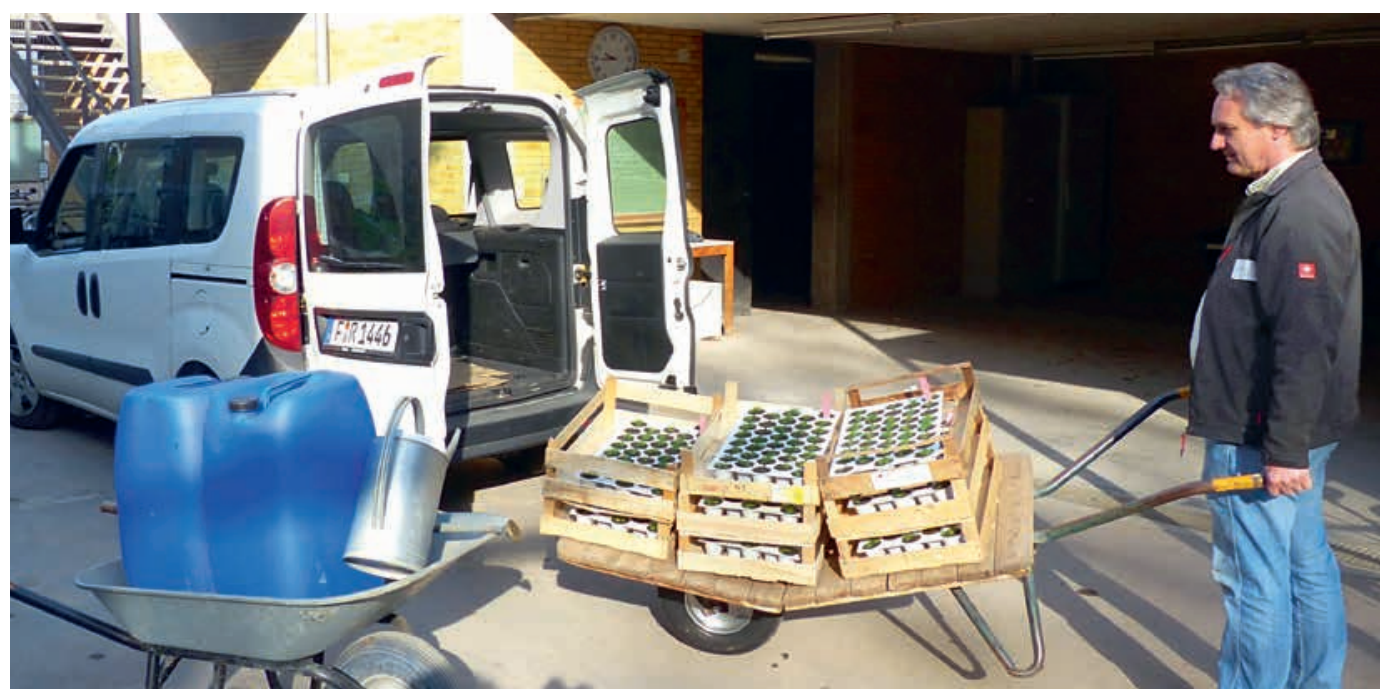

Abb. 1: Für ein Auswilderungsprojekt werden mehrere hundert Pflanzen (hier in Multitopfpaletten zu sehen) benötigt, dazu hundert Liter Wasser, Werkzeug, Gießkannen und Schubkarre - da wird der Wagen oft voll bis unters Dach! (Foto: U. BARTH) 


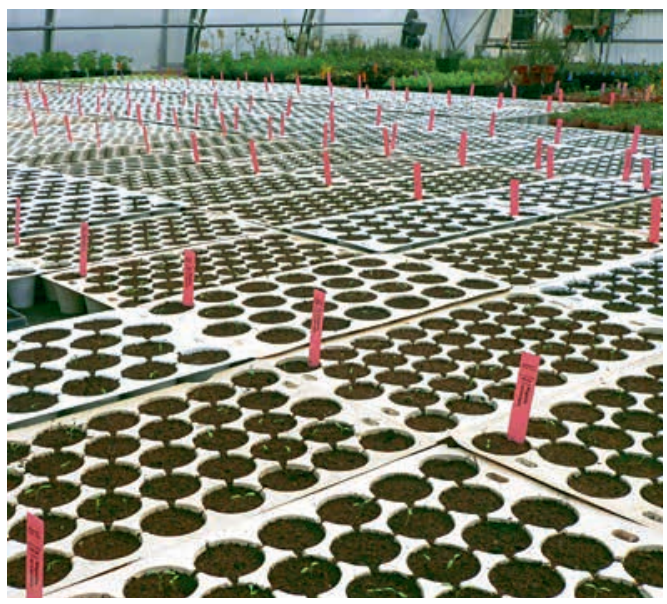

Abb. 2: Die Anzucht von Pflanzen in Multitopfplatten. (Foto: U. BARTH)

len“ und sogar „Arfurt an der Lahn“. Verwundert rieb sich mancher die Augen, denn es ist doch nicht Aufgabe eines Botanischen Gartens, seine Pflanzen in die heimische Natur zu verfrachten, oder etwa doch?

Nicht nur in weit entfernten Ländern, sondern auch in Deutschland sind Tier- und Pflanzenarten vom Aussterben bedroht. Manche der bedrohten Pflanzenarten sind bekannter als andere oder blühen besonders auffällig und schön. Doch auch die kleinen, oft unscheinbaren Arten haben ihren Stellenwert innerhalb ihres Lebensraumes, und mit jeder ausgestorbenen Art geht ein Stück Vielfalt verloren. Mit dem Schutz einiger dieser Pflanzenarten befasst sich ein Projekt, das am Botanischen Garten angesiedelt ist und von der KfW-Stiftung fünf Jahre lang gefördert wurde.

Botanische Gärten sind spätestens seit Beschluss des „Übereinkommens über die biologische Vielfalt" (Convention on Biological Diversity, CBD) von Rio de Janeiro 1992 wichtige Partner bei der Umsetzung internationaler Artenschutzziele. Im Rahmen der CBD wurde unter federführender Mitwirkung des Verbandes Botanischer Gärten eine spezifische Strategie zum Schutz von Wildpflanzen entwickelt. Mit dieser Globalen Strategie zum Schutz der Pflanzen (Global Strategy for Plant Conservation, GSPC) ist Deutschland völkerrechtliche Verpflichtungen

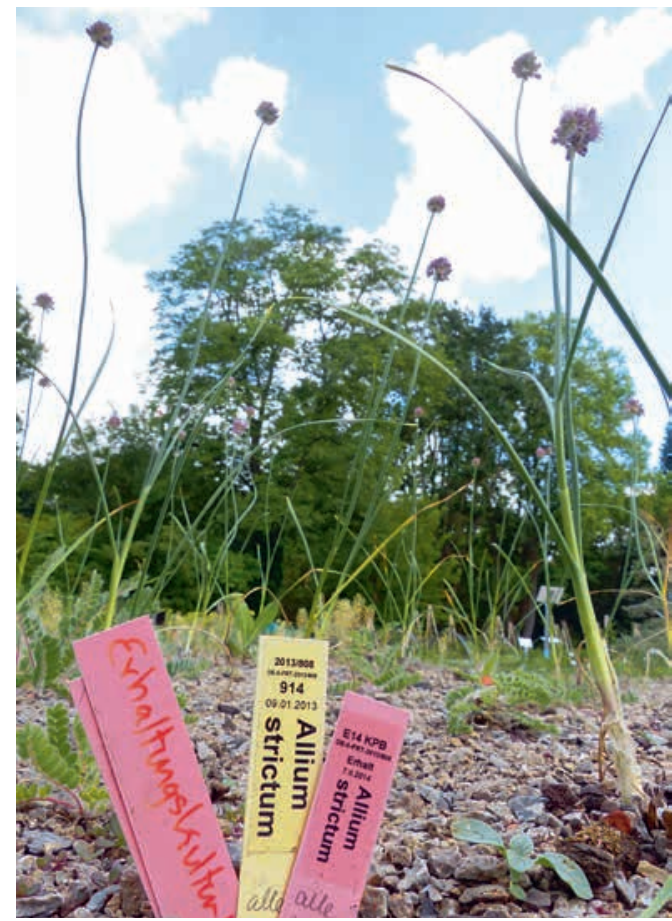

Abb. 3: Spezielles steiniges Substrat in einem Kulturbeet, hier mit blühendem Steifem Lauch (Allium strictum). Artspezifisch geeignete Kulturbedingungen sind Voraussetzung für eine erfolgreiche Erhaltungskultur. (Foto: U. BARTH)

eingegangen. Gemeinsames Ziel der Vertragsstaaten ist der Schutz der globalen biologischen Vielfalt (Biodiversität), speziell deren Erforschung, Erhaltung und nachhaltige Nutzung. Unter Biodiversität versteht man unter anderem die Vielfalt der genetischen Information, der Arten und der Ökosysteme. Ein wichtiger Aspekt der Strategie ist, dass der Schutz gefährdeter Arten sowohl am natürlichen Standort (in situ) als auch in menschlicher Obhut (ex situ) erfolgen soll.

Ein GSPC-Schutzziel ist, $75 \%$ der gefährdeten heimischen Pflanzenarten bis zum Jahr 2020 in ex-situ-Kulturen aufzunehmen und $20 \%$ für Wiederansiedlungsmaßnahmen zur Verfügung zu stellen. Die Kernkompetenz der Botanischen Gärten liegt in der Kombination von wissenschaftlicher Kenntnis und der gärtnerischen Fähigkeit, Wildpflanzen kultivieren zu können. Der Botanische Garten Frankfurt am Main hat schon seit ca. 1970 einen Sammlungsschwerpunkt bei der 


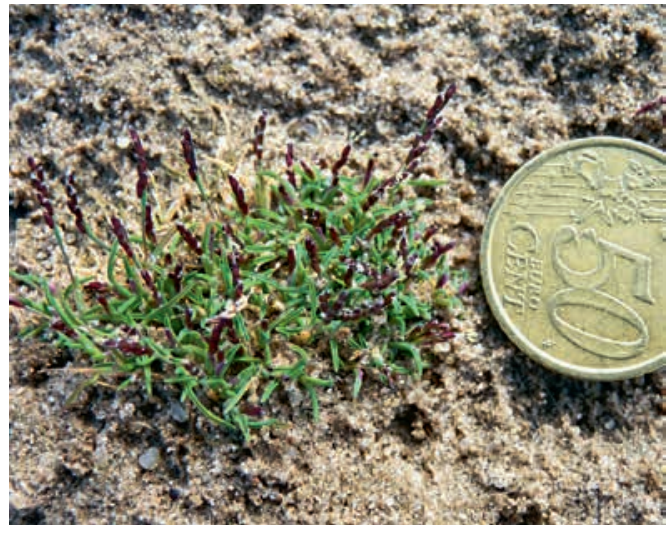

Abb. 4: Ein junger Büschel Sand-Zwerggras (Mibora minima) mit einer Münze als Größenvergleich. Vor der Blütezeit sind die Halme noch nicht in die Länge gestreckt, wodurch die Pflanzen recht unscheinbar aussehen. (Foto: U. BARTH)

Kultur gefährdeter einheimischer Pflanzenarten (GrasmücK 1996).

\section{Was ist eine Erhaltungskultur?}

Im praktischen Naturschutz gewinnen ex situ-Maßnahmen zunehmend an Bedeutung, und zwar als Ergänzung zur Pflege und Wiederherstellung von Lebensräumen. Viele der sehr selten gewordenen Arten haben in der heutigen Landschaft keine Möglichkeit mehr, aus eigener Kraft geeignete neue Flächen zu besiedeln. Der traditionelle Ansatz, nur Schutzgebiete auszuweisen und zu pflegen, reicht also nicht mehr aus. Dynamischere Strategien müssen entwickelt und mit angemessenen ex situ-Maßnahmen kombiniert werden. Keinesfalls jedoch dürfen sie ein Alibi sein für fehlende in situ-Maßnahmen (Biotoppflege) und nie zu Lasten anderer Schutzmaßnahmen erfolgen. Im Naturschutz und zur Erhaltung pflanzengenetischer Ressourcen wird daher heute ein integrativer Ansatz verfolgt, der in situ- und ex situ-Maßnahmen beinhaltet.

Besuchern des Botanischen Gartens ist vielleicht aufgefallen, dass im Bereich der ehemaligen Systematik neue Beete angelegt wurden, von denen jedes ein spezielles Substrat aufweist (Abb. 3), zum Beispiel Kalkschotter oder kalkfreier Sand. Hier findet man einen Teil unserer Erhaltungskulturen. Eine Erhaltungskultur ist eine Population einer Pflanzenart oder -unterart aus einer dokumentier- ten heimischen Wildherkunft, die in einem Garten kultiviert wird. Das Ziel der Erhaltungskultur ist es, das lokale, regionale oder globale Aussterben der jeweiligen Art zu verhindern. Aber wie funktioniert das praktisch? Mit diesem Artikel soll Licht in Dunkel gebracht und erläutert werden, was der Botanische Garten zum GSPC-Schutzziel beiträgt.

Im Zuge der Umsetzung der nationalen Strategie zum Schutz der biologischen Vielfalt wurde 2014 das bundesweite Projekt WIPs-De ins Leben gerufen. Dessen Ziel ist der „Aufbau eines nationalen Netzwerkes zum Schutz gefährdeter Wildpflanzenarten in besonderer Verantwortung Deutschlands". Aufgrund der eben geschilderten Erkenntnis werden im Projekt drei bisher meist isolierte Erhaltungsstrategien miteinander verknüpft:

a) Sammlung von Samen und Archivierung in Saatgutbanken;

b) Erhaltungs- und Vermehrungskulturen in Botanischen Gärten;

c) Wiederansiedlungen oder Stärkungen der Populationen an natürlichen Standorten.

\section{Die Pflanzenarten des Projektes}

Unser Projekt „Erhaltungskulturen“ entwickelte sich zeitgleich wie WIPs-De, ist aber regional auf Hessen bezogen. Zusammen mit der Botanischen Vereinigung für Naturschutz in Hessen e. V. (BVNH), die das Projekt seit Beginn fachlich begleitet und unterstützt, haben wir 15 Arten ermittelt, für die Hessen eine besondere Verantwortung trägt und für die Erhaltungskulturen aufgebaut werden sollen:

- Allium strictum (Steifer Lauch) - $\$$

- Festuca albensis (Tomans Schwingel; syn. F. tomanii, F. duvalii p.p.)

- Fumana procumbens (Gewöhnliches Nadelröschen)

- Iris spuria (Wiesen-Schwertlilie) - $\$$

- Jurinea cyanoides (Sand-Silberscharte) - $\$ \$, F F H$

- Mibora minima (Sand-Zwerggras)

- Moenchia erecta (Aufrechte Weißmiere) 


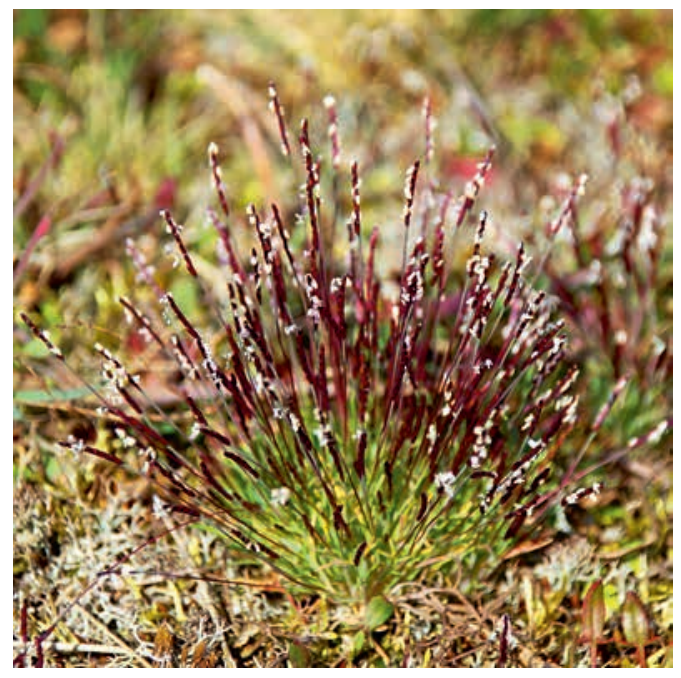

Abb. 5: Sand-Zwerggras (Mibora minima) in voller Blüte am 18. März. (Foto: S. Dietmann)

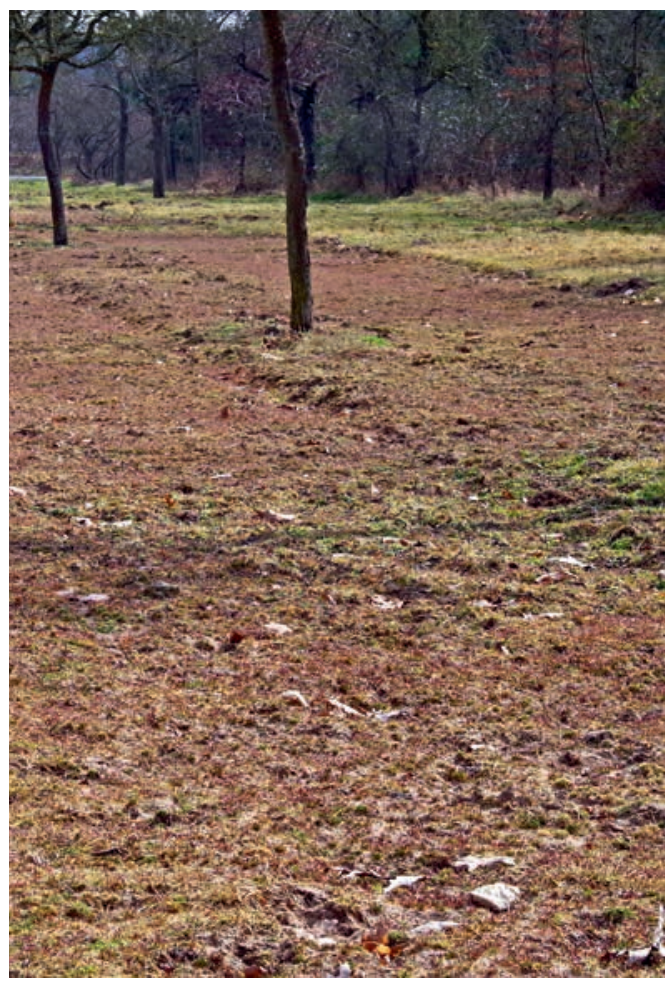

Abb. 6: Acker mit Streuobst am 18. März, durch blühendes Zwerggras rötlich gefärbt. Ein solches Bild ist nur unter Voraussetzungen möglich, die moderne Landwirtschaft praktisch nicht bieten kann: Die Fläche wurde im Hochsommer flach gegrubbert, (tiefes) Pflügen ist schädlich. Ab der Keimung im September kann sich das Zwerggras dann ungestört entwickeln. (Foto: S. DietmanN)
- Nigella arvensis (Acker-Schwarzkümmel)

- Poa badensis (Badener Rispengras)

- Scleranthus verticillatus (Hügel-Knäuelkraut)

- Sedum villosum (Sumpf-Fetthenne)

- Spergula pentandra (Fünfmänniger Spergel)

- Ventenata dubia (Zweifelhafter Grannenhafer)

- Veronica acinifolia (Drüsiger Ehrenpreis)

- Vicia orobus (Heide-Wicke)

Alle 15 Arten sind als „Verantwortungsarten“ Bestandteil der hessischen Biodiversitätsstrategie; drei sind gemäß Bundesartenschutzverordnung geschützt $(\$)$ bzw. streng geschützt $(\$ \$)$; die Sand-Silberscharte steht zudem auf Anhang II und IV der Flora-Fauna-Habitat-Richtlinie (FFH) der EU. Zwei dieser Arten sollen beispielhaft näher vorstellt werden. Es wird erklärt, warum wir uns um sie kümmern und was wir mit ihnen machen.

\subsection{Das Sand-Zwerggras (Mibora minima)}

Diese zierliche Pflanze ist einjährig, die Samen keimen im Herbst. Die höchstens $10 \mathrm{~cm}$ hohen Pflanzen sind über den Winter grün und blühen oft schon im Dezember (Abb. 4, 5). Das Zwerggras verträgt keine harten Fröste und ist daher auf wintermilde Gebiete beschränkt. Es vermehrt sich sexuell durch Windbestäubung, weshalb ausreichend große Populationen erforderlich sind, um ein Überleben langfristig zu gewährleisten (Abb. 6). Die Art besiedelt kalkfreie, trockene und nährstoffarme Sandböden.

Das Areal umfasst im Wesentlichen die Küsten Nordwest-Spaniens und West-Frankreichs. In Deutschland befindet sich ein wichtiges Binnenlandvorkommen im Rhein-Main-Gebiet. In Deutschland wie auch in Hessen gilt das Sand-Zwerggras als stark gefährdet. Die Verantwortlichkeit Hessens ist sehr hoch, denn es gibt im übrigen Deutschland nur noch wenige kleine Vorkommen, zum Beispiel bei Stockstadt am Main (Bayern) und bei Ingelheim am Rhein (Rheinland-Pfalz).

Das Verbreitungsgebiet umfasste früher fast die ganze Rhein-Main-Tiefebene (Abb. 7). Nach Gärtner et al. (1799) war die Pflanze ehemals „auf allen sandigen Aekkern und Feldern so häufig, $\mathrm{da} ß$ sie oft von ferne ganz roth davon scheinen, 


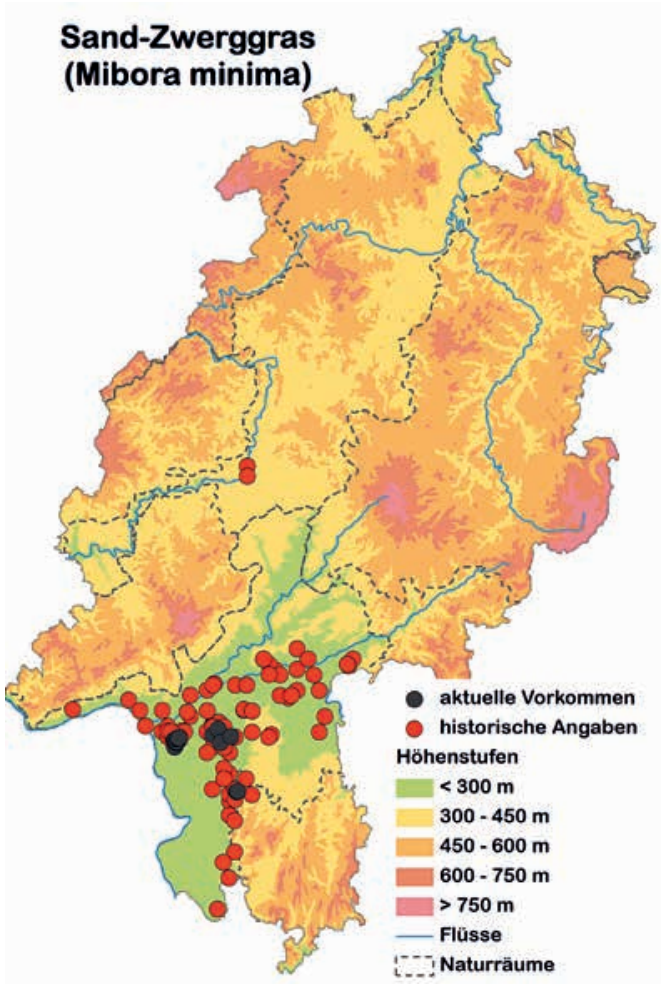

Abb.7: Das Sand-Zwerggras in Hessen. (Grafik: U. BARTH)

z. B. um Hanau, Offenbach, Enkheim, Bergen, Frankfurt, Giesen, Darmstadt etc. "In der Artendatenbank des Landes Hessen finden sich 241 Meldungen, die älteste aus dem Jahr 1713 (Hessisches Landesamt für Umwelt, Geologie und Naturschutz 2015).

Die aktuelle Situation sieht dagegen sehr düster aus: Demnach gibt es nur noch im Raum Rüsselsheim große Populationen, wie in Abb. 6 zu sehen, und die Bestände sind seit Jahren dramatisch rückläufig. Hodvina \& Buttleer (2002) berichten noch von gut 10 Millionen Individuen an 64 Standorten, aber im Jahr 2015 (DiETMANN 2015) waren es nur noch knapp 4 Millionen an 19 Standorten, und $99 \%$ der Pflanzen wuchsen an nur zwei Stellen! Eine Kontrolle der Vorkommen bei Mörfelden-Waldorf durch einen von uns beauftragten Botaniker ergab im März 2016, dass diese infolge Brache und Überbauung vollständig erloschen sind: „An keinem der früher notierten Wuchsorte ist die Art noch vorhanden.“ (ButTLER,

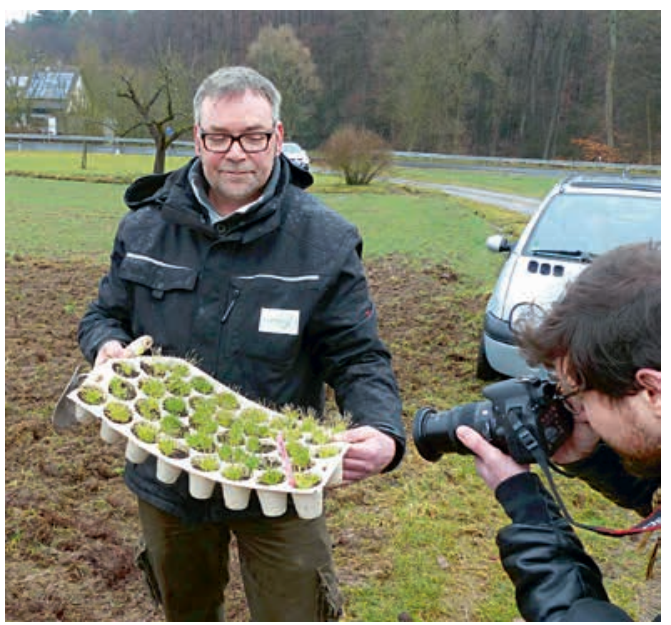

Abb. 8: Bei unserer ersten Wiederansiedlung haben wir Zwerggras sowohl gepflanzt als auch gesät und den Erfolg verglichen; die Pflanzung zeigte ein besseres Resultat, ist aber deutlich aufwändiger. (Foto: U. BARTH)

schriftl. 9. April 2016). Die konkurrenzschwache Pflanze ist nicht in der Lage, auf landwirtschaftlich intensiv genutzten Flächen zu überleben. Erschwerend kommt hinzu, dass keine langlebige Samenbank aufgebaut wird, sodass ungünstige Bedingungen nicht überbrückt werden können und die Populationen dann zusammenbrechen. Typische Wuchsorte sind lückige Scherrasen, offene Baumscheiben in extensiv genutztem Streuobst oder Sandrasenfragmente.

Aus den Erfahrungen mit unterschiedlichen Nutzungen und Pflegeformen sind wir über die Ansprüche der Art inzwischen gut informiert. So erfolgen in mehreren Gebieten mit Zwerggras-Vorkommen gezielte Pflegemaßnahmen, um die Habitatverhältnisse entsprechend zu optimieren. Ein Beispiel ist der Westteil der Apfelbachdüne bei Weiterstadt-Gräfenhausen, wo im Rahmen eines Restitutions- und Forschungsprojektes seit einigen Jahren mit Eseln beweidet wird. Dies schadet der Zwerggras-Population nicht, es werden lediglich einzelne Exemplare zertreten oder herausgerissen. Die durch den Tritt entstehenden offenen Bodenstellen sind aber geeignet als Keimbett für die Samen. Ein anderes Beispiel sind Streuobstäcker bei Rüsselsheim-Königstädten, wo die NABU-Sektion Rüsselsheim seit 2013 


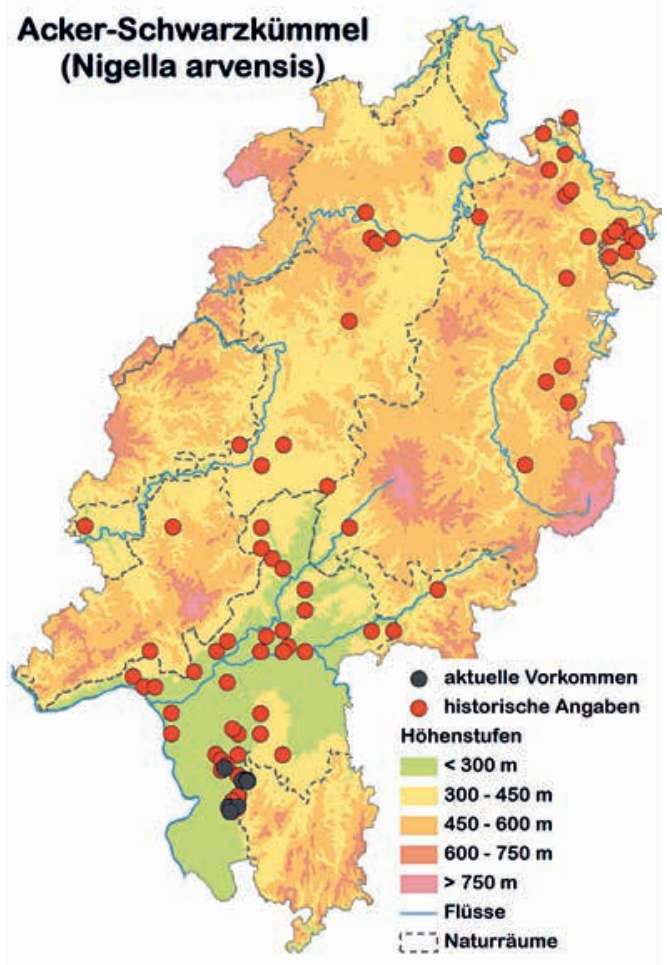

Abb. 9: Der Acker-Schwarzkümmel (Nigella arvensis) in Hessen. (Grafik: U. BARTH)

zusammen mit der Unteren Naturschutzbehörde Maßnahmen zum Erhalt des Zwerggrases durchführt, indem die sich schließende Vegetationsdecke periodisch im Spätsommer durch Fräsen gelockert oder partiell entfernt wird (SANDER, mündliche Mitteilung).

Unsere Erfahrungen aus der Kultur des Sand-Zwerggrases zeigen, dass die Samen im Herbst unter kühlen und feuchten Bedingungen keimen, und zwar am besten an der Oberfläche sandiger Böden. Aufgrund des unkomplizierten Keimverhaltens lässt sich die Art sehr leicht kultivieren, und sie eignet sich sehr gut für Ansiedlungsmaßnahmen, sowohl mit Samen als auch mit Pflanzen. Insofern lag es nahe, mit dem Sand-Zwerggras bereits im Februar 2015 unser erstes Auswilderungsvorhaben durchzuführen (Abb. 8).

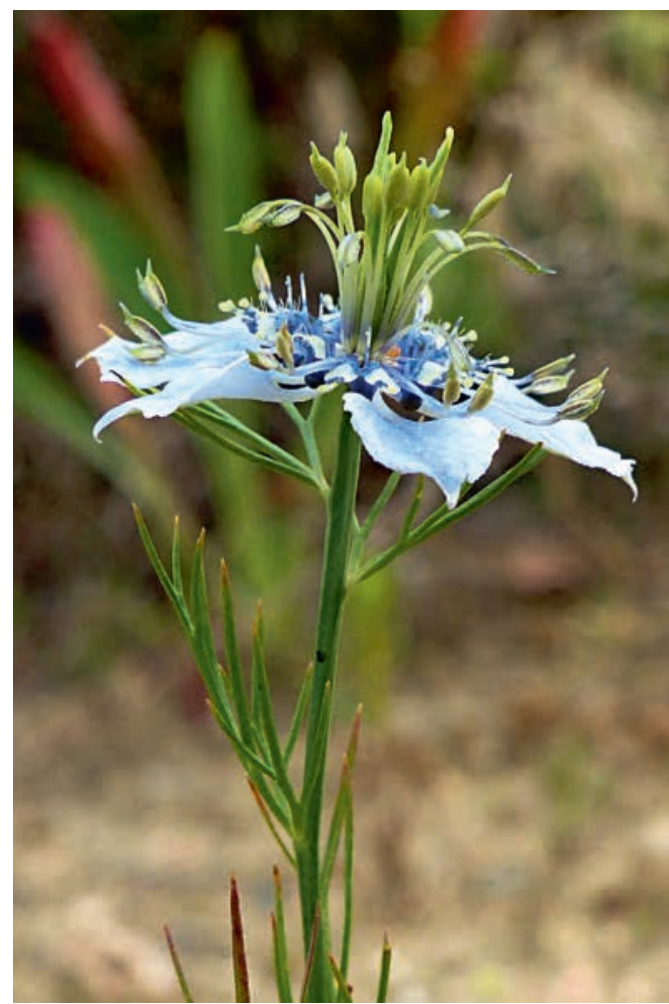

Abb. 10: Die attraktive Blüte des Acker-Schwarzkümmels (Nigella arvensis). (Foto: U. BARTH)

\subsection{Der Acker-Schwarzkümmel (Nigella arvensis)}

Diese sommerannuelle Pflanze mit attraktiver Blüte (Abb. 10) vermehrt sich vorwiegend mittels Insektenbestäubung (Nektarblume). Die Samen sind Dunkelkeimer und keimen im Frühling bei ausreichender Wärme. Nigella arvensis bildet eine langlebige Samenbank aus. Es handelt sich um ein archäophytisches Ackerwildkraut; der früheste Nachweis in Deutschland stammt aus dem Jahr 180 (römische Kaiserzeit). Die Pflanze besiedelt kalkhaltige Böden an trockenen Standorten.

Das Areal reicht von Westeuropa bis zum Schwarzen Meer; Deutschland liegt am nördlichen Arealrand. Die westlichsten Vorkommen (in Frankreich) und südlichsten (in Italien) sind größtenteils erloschen. Die Art ist im Gesamtareal nur mäßig häufig und zeigt deutliche Rückgangstendenz; sie ist europaweit stark gefährdet und in Hessen vom Aussterben bedroht. 
In der Artendatenbank des Landes Hessen liegen 203 historische Meldungen vor, die älteste von 1719 (HLNUG, 2015). Die Vorkommen des Acker-Schwarzkümmels beschränkten sich demnach keineswegs auf Südhessen, vielmehr ist auch ein ehemaliger Verbreitungsschwerpunkt in den Kalkgebieten von Nordosthessen erkennbar, wie die Karte in Abb. 9 verdeutlicht. Die hessischen Reliktvorkommen auf kalkhaltigen Sandböden bilden überregional eine Ausnahme; normalerweise bevorzugt der Acker-Schwarzkümmel Kalkscherbenäcker in warmen Lagen. Ehemals befanden sich fast alle hessischen Vorkommen auf solchen Kalkstandorten; sie sind dort jedoch aufgrund der landwirtschaftlichen Intensivierung bzw. Aufgabe von Grenzertragsstandorten inzwischen vollständig verschwunden.

Aktuell ist die Situation alarmierend, denn es gibt in Hessen nur noch eine große Population. Nördlich des Mains ist die Art völlig verschwunden und die Gesamtverbreitung auf weniger als $5 \%$ geschrumpft. „Unter Berücksichtigung der ebenfalls beträchtlichen Verluste in den benachbarten Bundesländern sind die verbliebenen südhessischen Fundorte überaus bedeutsam für die Art in Deutschland“ (Hodvina \& Cezanne 2007). Inzwischen besiedelt der ehemals über fast ganz Hessen verbreitete Acker-Schwarzkümmel jetzt nur noch eine Fläche von etwa $2000 \mathrm{~m}^{2}$, wobei sich rund $90 \%$ aller Individuen in den Schutzgebieten „Dulbaum“ und „Ulvenberg“ befinden.

\section{Professionelle Saatgutsammlung}

Wie helfen wir nun den Pflanzen? Am Anfang steht eine fachgerechte Sammlung von Samen, und damit fangen die Probleme schon an: Diese Arten sind sehr selten. Aber wie kommen wir an diese Samen, wo genau befinden sich die letzten Populationen? Hier kommen uns erfahrene Botaniker zu Hilfe, die sich oft seit Jahrzehnten mit bedrohten Arten befassen.

Auch die Sammeltätigkeit selbst ist keineswegs trivial: Für die Entnahme von Samen aus Wildpopulationen gibt es Richtlinien, die ein internationales Expertengremium erarbeitet hat

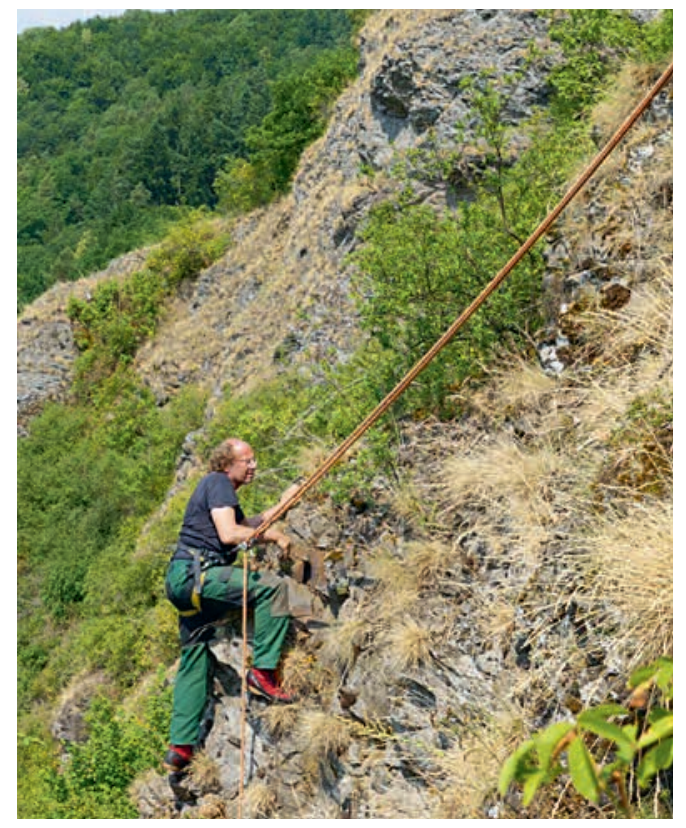

Abb. 11: Die Samensammlung beim Steifen Lauch (Allium strictum) war eine Herausforderung. (Foto: R. Кивоsсн)

(ENSCONET 2009). Diese bilden die fachliche Basis für die Tätigkeit der von uns beauftragten Artenkenner, die das Saatgut in der Natur sammeln. So ist einerseits gewährleistet, dass die Spenderpopulation durch die Saatgutentnahme nicht beeinträchtigt wird, und andererseits das gesammelte Saatgut den Qualitätsstandards entspricht: So sollten mindestens 30 Pflanzen einer Population besammelt werden, nur ausnahmsweise weniger, um das genetische Potenzial der Population möglichst vollständig zu erfassen. Zum Schutz der Spenderpopulation entnehmen wir nicht mehr als $10 \%$ der verfügbaren Samen; bei geschützten Arten oder in Schutzgebieten sogar nur max. $5 \%$. Die Sammlung erfolgt nach dem Zufallsprinzip; die gezielte Auswahl von Individuen aufgrund ihres Aussehens soll vermieden werden.

Samen ohne Dokumentation ihrer Herkunft sind für unsere Zwecke wertlos, daher ist jede Aufsammlung sorgfältig zu dokumentieren. Ein Sammelprotokoll enthält Angaben zu Sammelort, Populationsgröße und Umfang der Entnahme. Entsprechend der Lebensweise unserer Projektpflanzen erfordert die Saatgutsammlung 


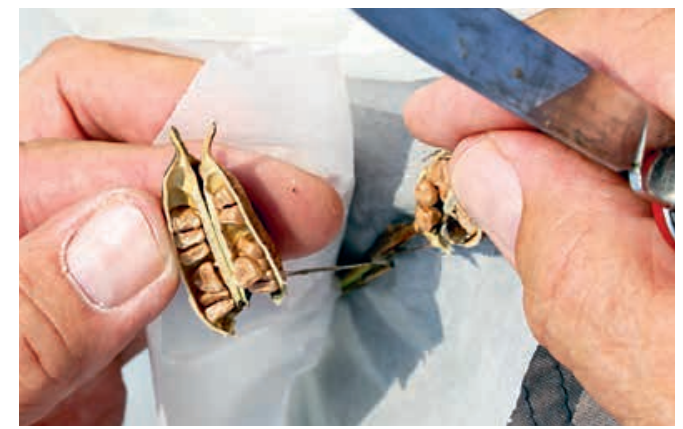

Abb. 12: Bereits im Gelände nehmen die Saatgutsammler erste Qualitätskontrollen vor, um das Einsammeln von parasitierten oder tauben Samen zu vermeiden.

(Foto: M. Hiemann)

mitunter beträchtlichen Einsatz: Abb. 11 zeigt einen von uns beauftragten Kletterprofi beim Besammeln des Steifen Lauchs (Allium strictum) in einer Felswand.

\section{Die Kultivierung der Pflanzen}

Die Kultur von Wildpflanzen in Botanischen Gärten mit dem Ziel der Wiederausbringung in der Natur wird zurecht mit Skepsis seitens der
Naturschutzverbände und -behörden betrachtet, denn genetische Vielfalt kann unter natürlichen Bedingungen am besten erhalten werden. Ein weiterer berechtigter Kritikpunkt ist, dass mit der Erhaltung von Pflanzen in Botanischen Gärten oder in Gen- und Samenbanken ein falsches „Sicherheitsgefühl“ erzeugt wird. Inzwischen wächst jedoch die Akzeptanz für die Notwendigkeit von Erhaltungskulturen und es gibt hierfür allgemein akzeptierte internationale Richtlinien bzw. Qualitätsstandards (IUCN 2014, LAUTERBACH et al. 2015). Ein wesentliches Qualitätskriterium für ex situ-Kulturen ist deren sorgfältige Durchführung und Dokumentation bis hin zur Auswilderung. Selbstverständlich erfolgt eine regelmäßige Überwachung der Kulturen in Hinblick auf Substrateignung, Kulturbedingungen und Zustand der Pflanzen. Wesentliche Eckdaten und Beobachtungen werden in einem Kulturbuch festgehalten. Dabei notieren wir zum Beispiel Keimrate und Verluste sowie Auffälligkeiten in Wachstum und Blühverhalten.

Für die Aussaat werden in der Regel Tonschalen, Töpfe (Abb. 14) oder Multitopfplatten

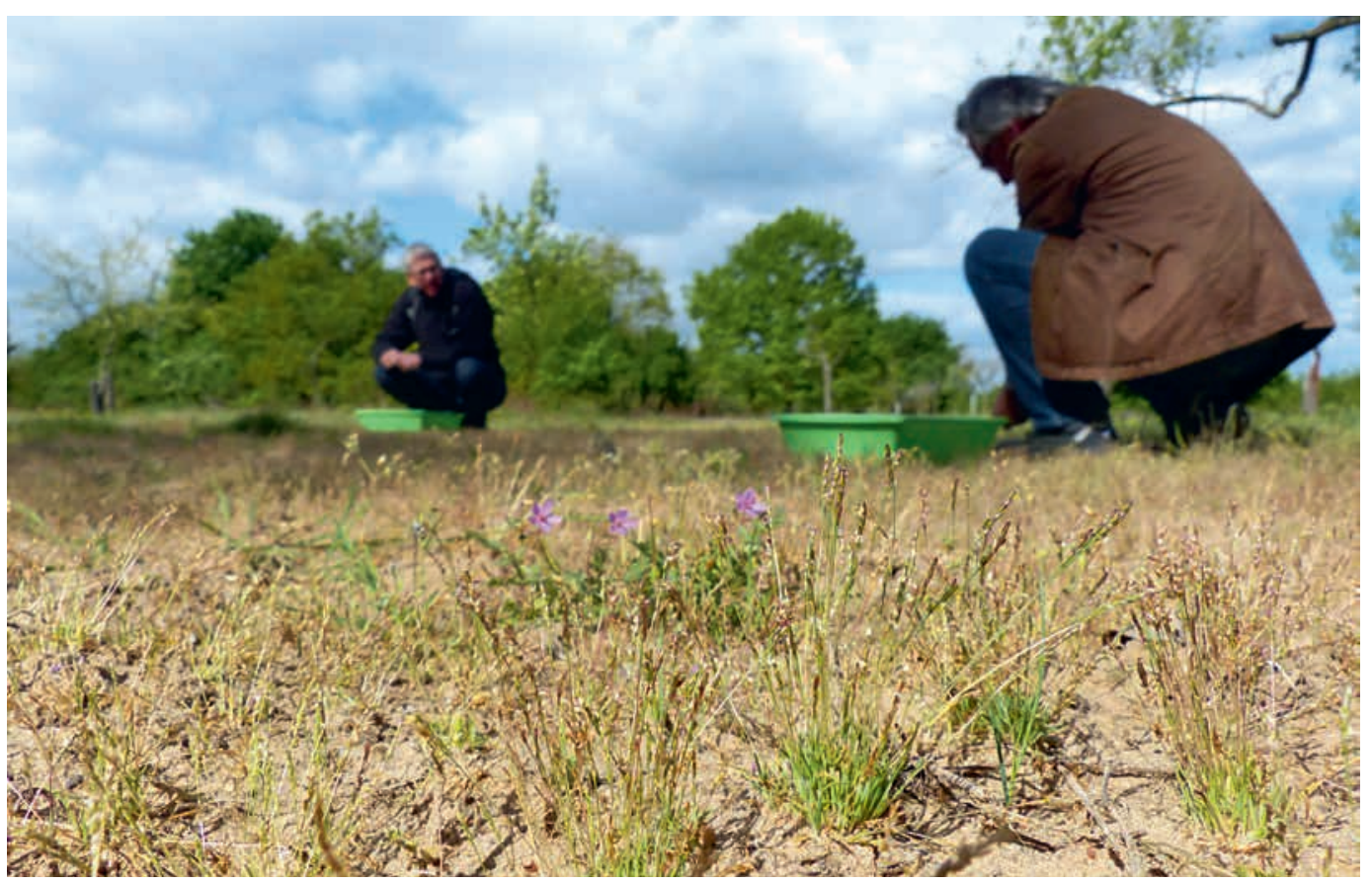

Abb. 13: Saatguternte in einer großen Population des Sand-Zwerggrases (Mibora minima). (Foto: U. BARTH) 


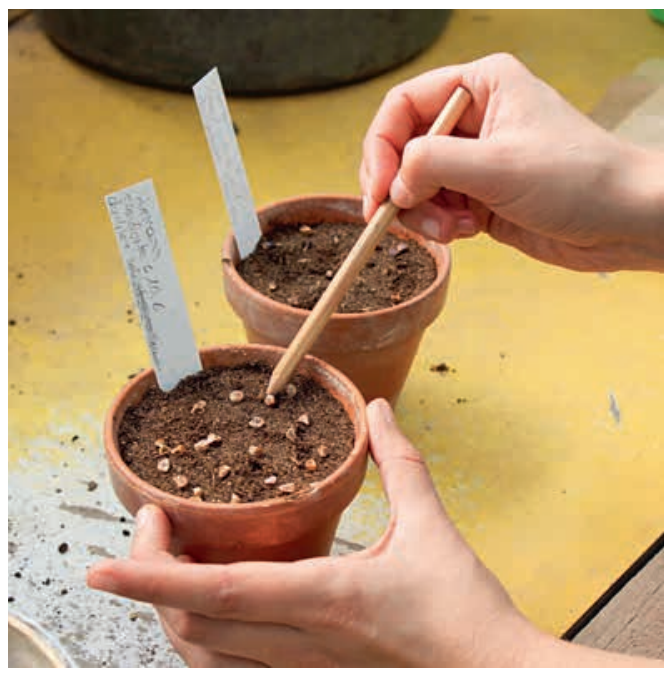

Abb. 14: Samen der Wiesen-Iris (Iris spuria) werden in Töpfe gesät. Die Samen sind abgezählt, damit die Keimrate ermittelt werden kann. (Foto: U. BARTH)

verwendet, wie sie in Abb. 2 zu sehen sind. Die Keimung erfolgt je nach Keimungstyp bei optimalen gärtnerischen Bedingungen unter Glas. Da es sich um heimische Pflanzen handelt, werden alle Arten anschließend unter annähernd dem Freiland entsprechenden Bedingungen angezogen. Die weitere Kultur der Arten bis zum Zeitpunkt der Ausbringung erfolgt unter Bedingungen, die den Anforderungen der jeweiligen Arten entsprechen. Dabei werden vor allem die unterschiedlichen Ansprüche hinsichtlich Substrat und Wasserversorgung berücksichtigt.

Die Grenzen einer ex situ-Kultur beschreiben KLingenstein et al. (2002) anschaulich, so dass die Autoren Botanische Gärten eher als zeitlich befristete „Intensivstation “ für bedrohte Arten betrachten, aber nicht als dauerhafte Heimat. Der am häufigsten beobachtete Schwachpunkt ist eine geringere genetische Diversität in Kultur und damit schlechtere Anpassungsmöglichkeiten an natürliche Verhältnisse.

Um solche Probleme zu vermeiden, bringen wir die aus dem zuvor in der Natur gesammelten Saatgut angezogenen Pflanzen oder deren Samen - also die so genannte F1-Generation - zurück in die Natur (rein-raus-Prinzip).

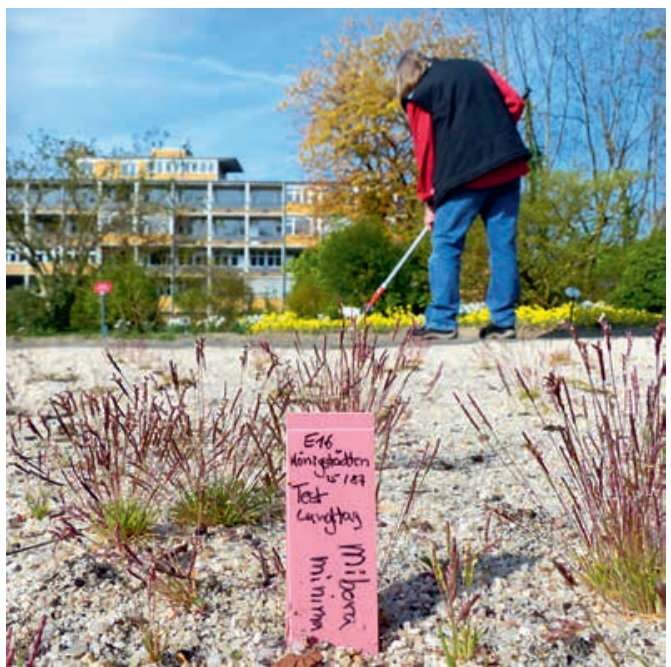

Abb. 15: Erhaltungskultur des Sand-Zwerggrases (Mibora minima) in einem Beet mit kalkfreien Sand im Botanischen Garten am 5. April 2017. (Foto: U. BARTH)

Außerdem verwenden wir für längerfristige Erhaltungskulturen eigens angelegte edaphisch-ökologische Beete (Abb. 3 und 15) und Biotopanlagen, in denen die Pflanzen unter naturnahen Bedingungen wachsen, also mit Konkurrenz und ohne Bewässerung bei Trockenstress (Abb. 17). Eine gärtnerisch optimierte Topfkultur ist zwar praktischer und bietet eine kontinuierlich günstige Wasser- und Nährstoffversorgung; aber unter solchen „Gartenbedingungen“ bringen jene Pflanzen einer Population die meisten Nachkommen

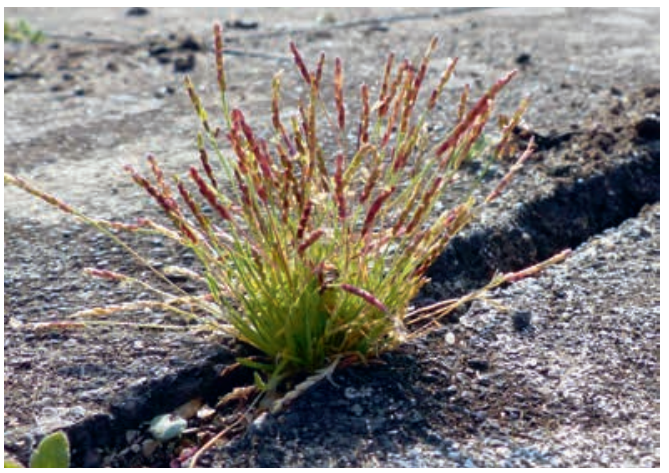

Abb. 16: Selbstaussaat von Sand-Zwerggras in der Pflasterritze eines Gartenweges. Hier ist eine besondere Einweisung bzw. Sachkenntnis des Gartenpersonals nötig, damit das seltene Gewächs nicht Reinigungsarbeiten zum Opfer fällt. Hier wurde die Fuge bereits ausgekratzt und unerwünschte Beikräuter sind entfernt. (Foto: U. BARTH) 


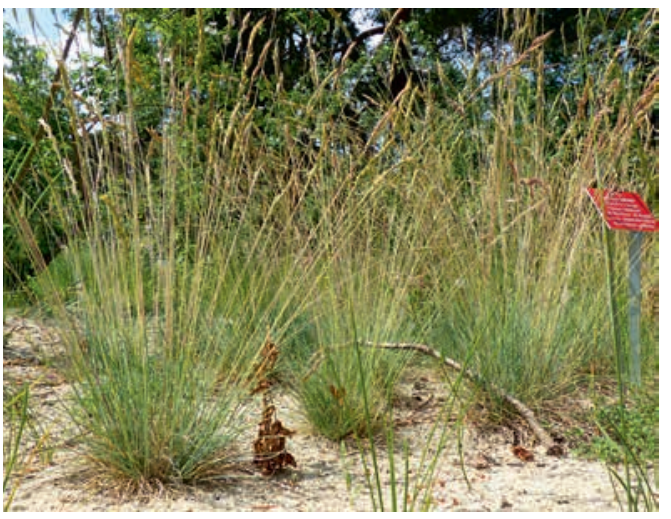

Abb. 17: Künstlich angelegte Sanddüne im Botanischen Garten mit einer Erhaltungskultur von Tomans Schwingel (Festuca albensis). Das Erscheinungsbild ähnelt der Situation am Naturstandort. Hier kann Selbstaussaat erfolgen und sollte im Idealfall den Fortbestand der Erhaltungskultur sichern. (Foto: U. BARTH)

hervor, die ein solches Optimum besonders gut ausnutzen können. In der Natur hingegen gibt es häufig weniger optimale Zustände, dagegen oft Stressphasen wie Dürre. Unter solch natürlichen Umständen produzieren die daran am besten angepassten Pflanzen der Population die meisten

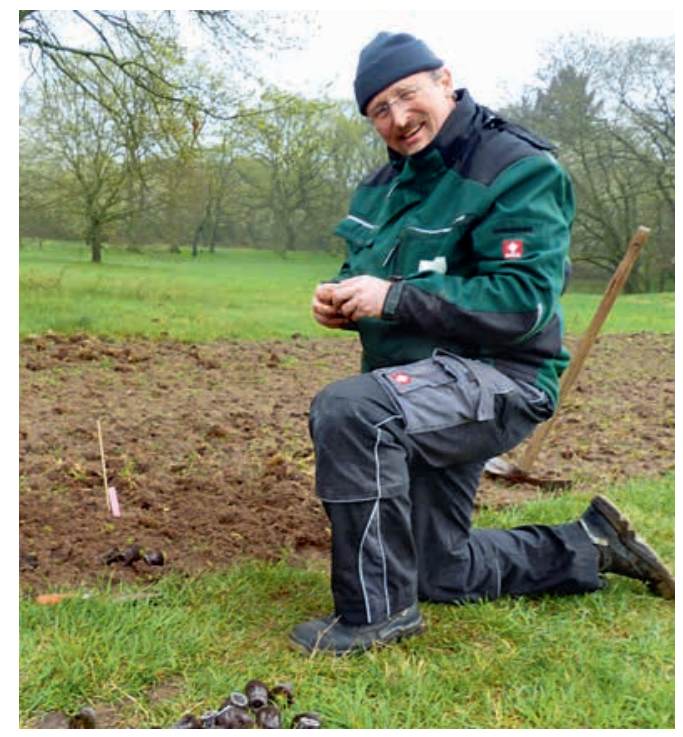

Abb. 18: Praktische Durchführung einer „leichten“ Auspflanzung in sandigem Untergrund, hier mit dem Sand-Zwerggras (Mibora minima) - da macht die Arbeit Spaß! Wichtig ist sowohl bei Pflanzung als auch bei Direktsaat eine geeignete Flächenvorbereitung, was hier mittels Grubbern des Oberbodens erfolgte. Vorn links die pflanzbereiten Zwerggraspflanzen als „Ballenware“. (Foto: U. BARTH)
Nachkommen, und das sind andere als die im Garten erfolgreichen Pflanzen. Eine Biotopkultur bietet Interaktionen mit anderen Arten, vor allem in Form von Konkurrenz, und ist daher am besten geeignet, die Lebensbedingungen am Naturstandort nachzubilden. Verschiedene Generationen stehen zusammen und die Keimung verläuft unter natürlichen Bedingungen.

\section{Zurück in die Natur}

Die bislang über vierzig Auswilderungsvorhaben verteilen sich ungleichmäßig auf unsere 15 Projektarten. Dies hat mehrere Gründe. Beim Sand-Zwerggras (Mibora minima) konnten wir dank großer Spenderpopulationen (Abb. 13) von Anfang an reichlich Saatgut sammeln, so dass dies auch die erste unserer Verantwortungsarten war, für die ein Wiederansiedlungsprojekt begonnen wurde.

Populationsstützungen (Ansiedlungen am Herkunftsort) spielen in unserem Projekt eine untergeordnete Rolle, weil aus nahe liegenden Gründen vorrangig große Populationen be-

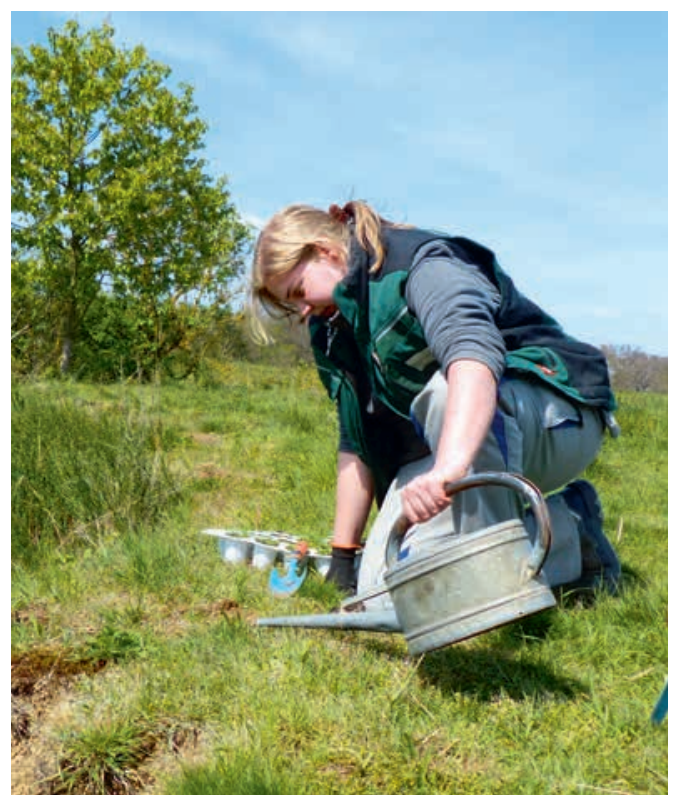

Abb. 19: Gründliches Angießen und eine minimale Nachsorge tragen zum Gelingen bei. Wenn man heimischen Wildpflanzen schon gärtnerisch unter die Arme greift, dann sollte dies auch nach allen Regeln der Kunst geschehen. (Foto: U. BARTH) 
sammelt wurden, die keiner Verstärkung bedürfen. Wir beschränken uns daher auf gezielte Ansiedlungen an aktuell unbesiedelten Stellen (Translokation). Grundlage unseres Handelns sind die internationalen Richtlinien für die Auswilderung von Pflanzen (IUCN 2013).

Mögliche Ansiedlungsorte müssen im (ehemaligen) Verbreitungsgebiet der jeweiligen Art liegen und für die jeweilige Art möglichst günstige Verhältnisse aufweisen. Die für den Rückgang der Art verantwortlichen Bedrohungen sollten am Ansiedlungsort nicht wirksam sein, sonst ist die Ansiedlung zum Scheitern verurteilt. Bei ehemaligen, erloschenen Vorkommen muss gesichert sein, dass der Aussterbegrund beseitigt ist. Sehr hilfreich sind dabei auch Kenntnisse von ortskundigen Gebietskennern, die oft über nicht publizierte Kenntnisse zum (ehemaligen) Vorkommen der jeweiligen Art verfügen.

Wenn ein geeigneter Ansiedlungsort gefunden ist, werden die Pflanzen oder Samen aber noch nicht gleich in die Freiheit entlassen, denn das Ausbringen von Pflanzen gebietsfremder Arten in der freien Natur bedarf gemäß $\$ 40$ Abs. 4 BNatSchG der Genehmigung der zuständigen Behörde. Künstlich vermehrte Pflanzen sind nur dann nicht gebietsfremd, wenn sie ihren genetischen Ursprung in dem betreffenden Gebiet haben. Als Gebiet wird dabei nicht eine streng begrenzte Fläche wie etwa ein Naturschutzgebiet verstanden, sondern eine naturräumliche Region (Landschaftseinheit), also zum Beispiel „Taunus“ oder „Wetterau“.

Wir führen ein Ansiedlungsvorhaben nur dann durch, wenn die Rahmenbedingungen positiv eingeschätzt werden, die Genehmigung erteilt ist und das Einverständnis des Flächeneigentümers vorliegt. Weil jede Zielfläche eine Kombination günstiger Bedingungen gleichzeitig erfüllen muss und alle Formalitäten geklärt sein müssen, reduziert dies die Zahl der realisierbaren Wiederansiedlungen enorm.

Da unsere Ansiedlungen im Vorfeld genehmigt sind und dokumentiert werden, handelt es sich

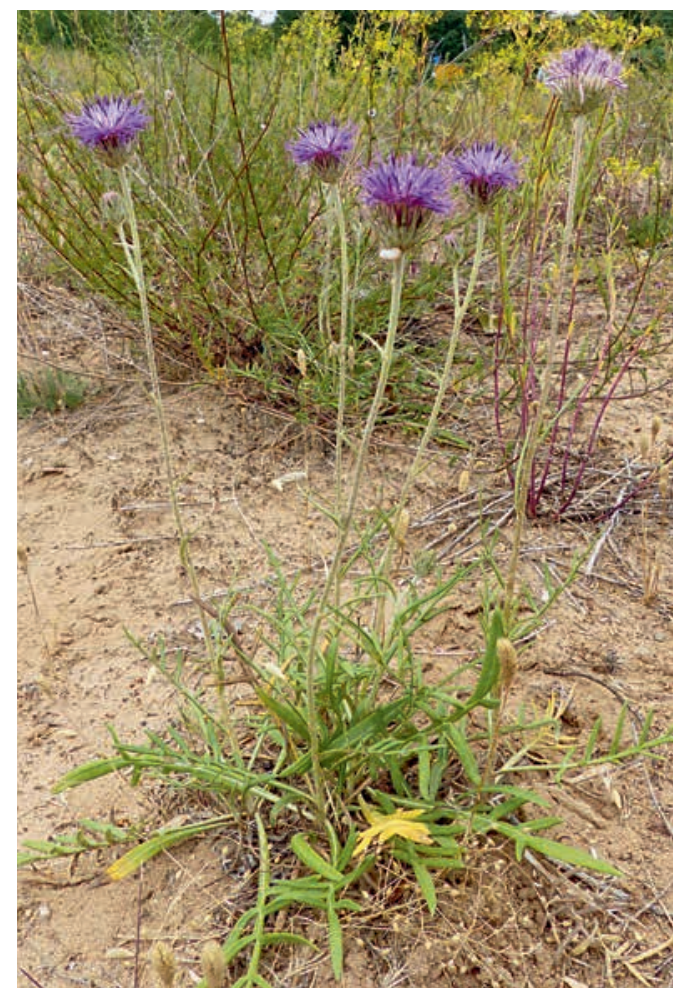

Abb. 20: Diese blühende Sand-Silberscharte (Jurinea cyanoides) stammt aus einer Anpflanzung zwei Jahre zuvor; die Überlebensrate der Pflanzen hier betrug rund $90 \%$. (Foto: U. BARTH)

auch nicht um so genannte „Ansalbungen“, wobei dieser Begriff oft im negativen Sinne benutzt wird. Denn Ansalbung ist definiert als „bewusstes Anpflanzen oder Aussäen einer Pflanze an einem Ort, wo sie nicht heimisch ist, als Versuch einer Einbürgerung ohne die Absicht einer Nutzung. Ansalbungen bedeuten eine Verfälschung der Natur und sind aus Naturschutzgründen abzulehnen, ganz besonders dann, wenn sie nicht bekannt gemacht werden“ (WAGENITZ 2008). Da unsere Pflanzen in Hessen bzw. in der jeweiligen Region (Naturraum) heimisch sind, trifft der so definierte Begriff nicht auf unsere Ansiedlungsprojekte zu.

Nicht selten müssen bei Pflanzungen hunderte Pflanzen über größere Strecken transportiert werden, und auch Gewicht und Menge des zum Angießen benötigten Wassers sind nicht zu unterschätzen. Aber wer einmal selbst mit eigenen 
Händen Hunderte Zwerggräser eingebuddelt hat, dem wird das Schicksal der Pflänzchen nicht egal sein! Solche Gemeinschaftsaktionen sind daher aus unserer Sicht eine ganz wichtige Investition in die Zukunft der neuen Populationen und dienen dazu, Interesse und Bewusstsein aufrecht zu erhalten. Unsere Wiederansiedlungen erfolgen deshalb immer öffentlich - entweder sind alle maßgeblichen Akteure anwesend, oder sie wurden zuvor über Sinn und Zweck des Vorhabens ausführlich unterrichtet. Nur wenn alle von der Sache überzeugt sind, wird das Vorhaben dauerhaft erfolgreich sein.

\section{Ausblick und Dank}

Über Erfolge und Misserfolge unserer Wiederansiedlungen werden wir im nächsten Jahr berichten; hierzu erfolgt eine Publikation in Botanik und $\mathrm{Na}-$ turschutz in Hessen, der Zeitschrift der BVNH. Für uns ist allein schon die bisherige Arbeit mit interessanten Pflanzen und engagierten Menschen ein schöner Erfolg. Eine von externen Experten durchgeführte Evaluation kommt zu einem guten Ergebnis und würdigt unser Projekt als vorbildlich. An dieser Stelle danken wir allen Beteiligten für Kooperation, Rat und Unterstützung, allen voran der KfW Stiftung für die Projektförderung. Wir werden uns dem Thema „Erhaltungskulturen“ natürlich weiterhin widmen. Zur Vertiefung des Themas „Wiederansiedlung von Pflanzen“ empfehlen wir die leicht lesbare Darstellung in der Broschüre von DiekMann (2016).

\section{Verwendete und weiterführende Literatur}

Diekmann, M: 2016: Handlungsleitfaden zur Wiedereinbürgerung von Pflanzenarten als Naturschutzmaßnahme. Unter Mitarbeit von Müller, J, Wittig, B. \& Dupré, C. Broschüre, herausgegeben von der Deutschen Bundesstiftung Umwelt (DBU).

Dietmann, S. 2015: Ermittlung und Untersuchung tatsächlicher und potenzieller Standorte des Zwerggrases Mibora mini$m a$ (L.) Desv. (Poaceae) mit physisch-geographischen Methoden im Rahmen eines Artenschutzprojektes. - Bachelorarbeit; J. W. Goethe Universität, Frankfurt am Main.

ENSCONET 2009: ENSCONET Anleitung zum Sammeln von Wildpflanzensamen. Deutsche Fassung des ENSCONET Seed collecting manual for wild species. - Kew
Gärtner, G.; Meyer, B.; Scherbius, J. 1799-1802: Oekonomisch-technische Flora der Wetterau. - Frankfurt am Main.

Grasmück, H. 1996: Erhaltungskultur gefährdeter Pflanzenarten im Botanischen Garten der Johann Wolfgang Goethe-Universität in Frankfurt am Main. - Geobot. Kolloq. 12: 31-36.

Hodvina, S. \& Buttler, K. P. 2002: Historische und aktuelle Verbreitung des Zwerggrases (Mibora minima) in Hessen. Bot. Natursch. Hessen 14: 91-118.

Hodvina, S. \& Cezanne, R. 2007: Der Acker-Schwarzkümmel (Nigella arvensis) in Hessen. - Bot. Natursch. Hessen 20: 61-88.

IUCN Species Survival Commision 2013: Guidelines for reintroductions and other conservation translocations. Version 1.0. International Union for Conservation of Nature. - Gland.

IUCN Species Survival Commision 2014: Guidelines on the use of ex situ management for species conservation. Version 2.0. International Union for Conservation of Nature. - Gland.

Klingenstein, F., van den Driesch, M. \& Lobin, W. 2002: Bedeutung und Aktivitäten der Botanischen Gärten im ex-situ- und in-situ-Artenschutz in Deutschland auf Grundlage der Biodiversitäts-Konvention. - Schriftenreihe für Vegetationskunde 36: 139-150.

Lauterbach, D., Borgmann, P., Daumann, J., Kuppinger, A.-L., Listl, D., Martens, A., Nick, P., Oevermann, S., Poschlod, P., Radkowitsch, A., Reisch, C., Stevens, A.-D., Straubinger, C., Zachgo, S., Zippel, E. \& Burkart, M. 2015: Allgemeine Qualitätsstandards für Erhaltungskulturen gefährdeter Wildpflanzen. - Gärtn.-Bot. Br. 200: 16-39.

Wagenitz, G. 2008: Wörterbuch der Botanik, 2. Aufl. Heidelberg.

\section{Internetseiten}

https://umwelt.hessen.de/umwelt-natur/naturschutz/hessische-biodiversitaetsstrategie

http://www.ex-situ-erhaltung.de

http://www.botanischergarten-frankfurt.de/der-garten/ erhaltungskulturen-gefaehrdeter-wildpflanzen/projekttagebuch-2018.html

https://www.dbu.de/doiLanding1423.html (DBU-Broschüre)

\section{Anschrift der Autoren:}

Uwe Barth \& Andreas König, Palmengarten, Botanischer Garten Frankfurt am Main, Siesmayerstr. 61, 60323 Frankfurt am Main, E-Mail: uwe.barth@stadt-frankfurt.de bzw. andreas.koenig@stadt-frankfurt.de 